\section{Equidade inversa e desigualdades no acesso à tecnologia no parto em Santa Catarina, Brasil, 2000 a 2004}

\section{Inverse equity and inequalities in the use of technology in childbirth, in Santa Catarina, Brazil, 2000 to 2004}

\begin{abstract}
Objectives: to investigate changes over time and factors associated with caesarean section rates in the State of Santa Catarina, Brazil, between 2000 and 2004.

Methods: data from the Live Births National Information System for the State of Santa Catarina were used. The variables analyzed were maternal age and literacy, ethnicity/skin color of the newborn, duration of gestation and number of prenatal consultations. Crude and adjusted prevalence rates were estimated for each of the variables using Poisson regression.

Results: rates for the period were three times higher of those accepted by the World Health Organization and increased from $43.3 \%$ in 2000 to $50.6 \%$ in 2004. For the whole period (2000-2004) prevalence rates, both crude and adjusted, were found to be positively associated with higher maternal literacy $\left(P R_{a d j}=1.50\right.$; 95\% CI: 1.471,52), older age $\left(P R_{a d j}=2.10 ; 95 \% C I: 2.05-2.15\right)$, greater number of prenatal consultations $\left(P R_{a j}=1.27\right.$; 95\%CI: 1.26-1.29), pre-term $\left(P R_{a d j}=1.10 ; 95 \% C I\right.$ : 1.06-1.13) and post-term deliveries $\left(P R_{a d j}=1.22\right.$; 95\% CI: 1.14-1.30), and protection for the indigenous $\left(P R_{a d j}=0.79 ;\right.$ 95\%CI: 0.75-0.85) and "non-white" newborns $\left(P R_{a d j}=1.10 ;\right.$ C195\%: 1.06-1.14). A significant decrease in the adjusted prevalence ratios (PRs) when comparing the extremes in the period (2000 and 2004) appeared for all variables and categories.

Conclusions: the caesarean section rates were well above those justified for medical reasons alone. The decrease in the caesarean section PRs when comparing extremes during the period calls attention to a reduction in the effects of the "inverse equity" probably connected to a higher access to childbirth technology among women of lower socio-economic status, at least partly attributable to more liberal use of this technology in obstetrical practice, including more widespread medical recommendation of caesarean section.
\end{abstract}

Key words Cesarean Section, Risk factors, Health inequalities, Socioeconomic factors, Ethnic groups, Educational status, Maternal age, Women's health, Indigenous population
Julio César de Souza Junior ${ }^{1}$

Nádia Kunkel ${ }^{2}$

Marcius de Almeida Gomes ${ }^{3}$

Paulo Fontoura Freitas 4

1-4 Programa de Pós-Graduação em Saúde Pública. Centro de Ciências da Saúde. Departamento de Saúde Pública. Universidade Federal de Santa Catarina. Campus Universitário Trindade. CEP: 88.010-970. Florianópolis, SC, Brasil.

E-mail: pfreitas@ccs.ufsc.br

\section{Resumo}

Objetivos: investigar a evolução temporal das taxas de cesariana e fatores associados em Santa Catarina, no período de 2000 a 2004.

Métodos: foram utilizados dados do Sistema de Informações de Nascidos Vivos. As variáveis analisadas foram: escolaridade, idade materna, etnia/cor da pele do recém-nascido, duração da gestação e número de consultas pré-natal. Razões de prevalência de cesariana, brutas e ajustadas para confundimento, foram estimadas para cada uma das variáveis utilizando Regressão de Poisson.

Resultados: as taxas encontradas para o período foram o triplo daquelas preconizadas pela Organização Mundial de Saúde e aumentaram de 43,3\% em 2000 para 50,6\% em 2004. Para todo o período razões de prevalência brutas e ajustadas mostraram-se positivamente associadas ao mais alto grau de escolaridade $\left(R_{P a j}=1,50\right.$; IC95\%: 1,47-1,52), idade mais elevada $\left(R_{P a j}=2,10\right.$; IC95\%: 2,05-2,15), maior freqüência ao pré-natal $\left(R P_{a j}=1,27 ;\right.$ IC95\%: 1,26-1,29), partos pré-termo $\left(R P_{a j}=1,10 ; I C 95 \%\right.$ : 1,06-1,13) e pós-termo ( $R P_{a j}=1,22$; IC95\%: 1,14$1,30)$ e proteção para as etnias "indígena" $\left(R P_{a j}=0,79 ;\right.$ IC95\%: 0,75-0,85) e "não branca" $\left(R P_{a j}=1,10\right.$; IC95\%: 1,06-1,14). Uma diminuição significante nas RPs ajustadas ao comparar os extremos do período (2000 e 2004) apareceu para quase todas as categorias estudadas.

Conclusões: as taxas de cesariana encontradas estão bem acima daquelas justificando indicações estritamente médicas. Uma diminuição nas RPs ao comparar os extremos do período nos remete a uma redução temporal nos efeitos da "equidade inversa", provável reflexo de um maior acesso à tecnologia no parto entre as camadas de menor padrão socioeconômico, pelo menos em parte atribuível a uma maior liberalidade da prática obstétrica, incluindo uma ampliação das indicações médicas.

Palavras-chave Cesárea, Fatores de Risco, Desigualdades em Saúde, Fatores socioeconômicos, Grupos étnicos, Escolaridade, Idade materna, Saúde da mulher, População indígena 


\section{Introdução}

A cesariana tem conhecido valor como procedimento para salvar vidas em determinadas e precisas indicações. ${ }^{1}$ Se a indicação correta de cesariana traz vantagens, sua indicação indiscriminada aumenta o risco de morbidade e mortalidade maternas e perinatal, além de elevar os custos quando comparada ao parto vaginal. ${ }^{2}$ Risco de prematuridade, em decorrência do uso não criterioso da tecnologia, como o ultra-som, falhas na determinação precisa da idade gestacional e associados com cesarianas decididas eletivamente têm sido sugeridos desde os anos 80 , tanto no Brasil $^{3}$ quanto internacionalmente. ${ }^{4}$ Os resultados de um estudo conduzido por Barros et al., ${ }^{5}$ em Pelotas, no Rio Grande do Sul, mostram que a prevalência de prematuridade cresceu de $6,3 \%$ em 1982, para 16,2\% em 2004. De acordo com esses autores, um aumento concomitante nas taxas de cesariana no mesmo período trazem à tona possíveis efeitos do abuso da tecnologia no parto.

Resultados de um amplo inquérito em Saúde Materna e Perinatal, conduzido recentemente em oito países da América Latina, ${ }^{6}$ mostram que a proporção de recém-nascidos com permanência maior do que sete dias, nas Unidades de Tratamento Intensivo, foi significativamente maior entre aqueles hospitais que apresentavam as maiores taxas de cesariana. Villar et al. ${ }^{6}$ sugerem que tais resultados podem refletir um aumento na ocorrência da síndrome da angústia respiratória do recém-nascido associado às cesarianas eletivas.

Apesar dos conhecidos riscos e dos limites preconizados pela World Health Organization (WHO), ${ }^{7}$ as taxas de cesariana vêm aumentando sistematicamente em vários países do mundo. ${ }^{8-10} \mathrm{O}$ Brasil apresenta uma das mais altas taxas mundiais, ${ }^{5}$ no ano de 2002, 38,7\% dos nascimentos no Brasil ocorreram por cesariana. No mesmo ano, as taxas para o Estado de Santa Catarina chegaram a $46,5 \% .^{11}$

Ainda que diferentes estratégias dirigidas ao problema das altas taxas de cesariana tenham sido $\operatorname{propostas}^{12,13}$ são raras as intervenções clínicas dirigidas a sua redução, e relatos de iniciativas na área praticamente inexistem na América Latina. ${ }^{8}$ Recentemente, uma intervenção conduzida por Althabe et al., ${ }^{14}$ em 36 hospitais de cinco países latino-americanos, testou a hipótese de que o uso de segunda opinião poderia reduzir as taxas de cesariana sem comprometer indicadores maternos e perinatais. Althabe et al. ${ }^{14}$ também verificaram que os resultados de uma redução bem abaixo do esperado podem ser atribuídos principalmente às dificuldades em mudar atitudes médicas, conclusão essa corroborada pela alta concordância entre os obstetras participantes da intervenção.

Sabendo-se que fatores médicos isoladamente não podem justificar o aumento nas taxas, torna-se de extrema importância o entendimento de tais fatores associados à cesariana. Entre os fatores de risco de consenso na literatura estão nível socioeconômico, alta escolaridade, maior número de consultas pré-natal, primiparidade, parto operatório prévio e parto privado. ${ }^{15-19}$

No Brasil, as altas taxas de cesarianas constituem um problema de saúde pública e nos remete às diversas formas de desigualdade social, representadas por taxas mais elevadas em mulheres potencialmente de menor risco obstétrico. Esses dados nos remetem ao princípio da "equidade inversa", de acordo com o qual uma diminuição nas desigualdades depende de um maior acesso das classes menos previlegiadas às intervenções ${ }^{20} \mathrm{e}$ sugerem um mau uso, ou até abuso, da tecnologia médica na atenção ao parto. ${ }^{5}$

O presente estudo teve como objetivo investigar os fatores associados à ocorrência de cesariana no Estado de Santa Catarina, no período de 2000 a 2004.

\section{Métodos}

Trata-se de um estudo com delineamento transversal que utilizou informação referente a 123.174 nascimentos hospitalares, de primíparas, com gravidez única. Foram utilizados dados do Sistema de Informações de Nascidos Vivos (SINASC) ${ }^{11}$ para o Estado de Santa Catarina, Paraná, Brasil, no período de 2000 a 2004. Recém-nascidos de multíparas não foram incluídos na análise uma vez que os dados do SINASC não contêm informação a respeito dos partos anteriores, impossibilitando assim o ajuste para cesariana prévia, fator de confundimento importante. Da mesma forma os partos domiciliares, por estarem associados com a probabilidade quase exclusiva de parto normal e, os partos múltiplos por se constituírem em indicação relativa para cesariana, foram excluídos.

As variáveis analisadas, como potencialmente associadas à ocorrência de cesariana, foram idade e escolaridade maternas, etnia/cor da pele do recémnascido, duração da gestação e freqüência ao prénatal.

Razões de prevalência de cesariana (RP), brutas e ajustadas para confundimento, foram estimadas para cada uma das variáveis de interesse utilizando 
Regressão de Poisson com variância robusta. Para as análises foi utilizado o pacote estatístico Stata versão 9.1. Modelos multivariados foram obtidos para cada ano do nascimento separadamente. A significância estatística das associações entre as variáveis estudadas e a prevalência de cesariana foi calculada no nível de significância de $95 \%$. Utilizou-se o teste do qui-quadrado $\left(\chi^{2}\right)$ para testar a existência de homogeneidade nas taxas de cesariana ao comparar categorias das variáveis independentes.

Os modelos multivariados seguem uma concepção teórica com dois níveis hierárquicos de influência. As variáveis idade e escolaridade maternas, etnia/cor da pele do recém-nascido e duração da gestação estão situados em um nível superior (nível 1) enquanto frequiência ao pré-natal é analisada como pertencente a um nível hierárquico de influência situado logo abaixo das outras variáveis e incluída posteriormente. A concepção teórica do modelo entende frequiência ao pré-natal como possível mediador da influência das outras variáveis na determinação final da cesariana, visto a maior probabilidade de decisões quanto à cesarianas eletivas serem tomadas como parte destas consultas. $^{21}$

Com exceção de etnia/cor da pele do recémnascido, para todas as outras variáveis as categorias escolhidas como referência foram os de menor riscos esperados (mãe analfabeta, com menos de 20 anos, 37 a 41 semanas de gestação, ausência de pré-natal). Para etnia/cor do recém-nascido, cor branca foi utilizada como referência, uma vez que etnia indígena, a categoria de menor risco, apresentava tamanho de amostra relativamente pequeno.

\section{Resultados}

O número de partos de nascidos vivos, no Estado de Santa Catarina durante o período de 2000 a 2004 foi de 439.104 , sendo que $44,4 \%$ ocorreram através de cesariana. Destes, 123.104 nascimentos ocorreram entre primíparas com gravidez única e parto hospitalar, correspondendo a $28 \%$ dos nascidos vivos no período, sendo $46,9 \%$ por cesariana. Os resultados mostram que as taxas de cesariana vêm crescendo nos últimos cinco anos, com um aumento estatisticamente significante $(p<0,01)$ de $43,3 \%$ em 2000 para 50,6\% em 2004. (Tabela 1).

A Tabela 1 mostra ainda que as categorias que apresentaram o maior risco de cesariana, ao longo do período, quando comparadas àquelas de menor risco foram idade maior de 35 anos, escolaridade maior de 12 anos completos e frequiência ao pré-natal de mais de sete consultas. Para etnia/cor da pele do recémnascido a maior proteção esteve associada à categoria etnica "não branca", quando comparada aos recém-nascidos da etnia "branca".

As taxas de cesariana apresentaram um aumento linear no período $(p<0,01)$ para todas as faixas de idade e escolaridade, para as duas categorias de frequência ao pré-natal e para as idades gestacionais pré-termo e pós-termo.

Os maiores aumentos nas taxas, ao comparar os extremos do período ocorreram entre as mulheres de 20 a 34 anos, as com menor escolaridade e aquelas que realizaram um menor número de consultas prénatal. Ainda, as taxas aumentaram proporcionalmente e de maneira importante para etnia/cor da pele do recém-nascido tanto branca, quanto negra, e indistintamente para todas as classes de idade gestacional.

Ao ajustar para o efeito de confundimento, como mostrado na Tabela 2, idade maior de 35 anos, escolaridade maior de 12 anos, freqüência ao pré-natal de mais de sete consultas e idade gestacional póstermo mantiveram-se associados de maneira significante com uma maior prevalência de cesarianas em todos os anos. Para idade gestacional menor de 37 semanas o efeito só não se manteve estatísticamente significante para o ano de 2004.

A Tabela 2 também mostra que uma diminuição significante nas RPs ajustadas $(p<0,05)$ ocorreu entre as mulheres com 35 anos ou mais e no grupo com maior número de consultas pré-natal, ao comparar extremos do período (2002 e 2004).

Para etnia/cor da pele, após o ajuste, o efeito protetor para a categoria "não branca" se mantém para todos os anos, exceto para 2002. Para etnia "indígena", ainda que o efeito protetor seja mais intenso, os resultados alcançaram significância estatística $(p<0,05)$ apenas ao analisar o período como um todo. 
Tabela 1

Valores absolutos, taxas e razões de prevalência brutas $\left(\mathrm{RP}_{\mathrm{b}}\right)$ de cesariana para as variáveis maternas e do recém- nascido em primíparas com gravidez única e parto hospitalar. Santa Catarina, 2000 a 2004.

\begin{tabular}{|c|c|c|c|c|c|c|c|c|c|c|c|c|c|c|c|}
\hline \multirow[b]{2}{*}{ Variáveis } & \multicolumn{3}{|c|}{2000} & \multicolumn{3}{|c|}{2001} & \multicolumn{3}{|c|}{2002} & \multicolumn{3}{|c|}{2003} & \multicolumn{3}{|c|}{2004} \\
\hline & $\mathrm{n}$ & $\begin{array}{c}\text { taxa } \\
\%\end{array}$ & $\mathrm{RP}_{\mathrm{b}}$ & $\mathrm{n}$ & $\begin{array}{c}\text { taxa } \\
\%\end{array}$ & $\mathrm{RP}_{\mathrm{b}}$ & $\mathrm{n}$ & $\begin{array}{c}\text { taxa } \\
\%\end{array}$ & $\mathrm{RP}_{\mathrm{b}}$ & $\mathrm{n}$ & $\begin{array}{c}\text { taxa } \\
\%\end{array}$ & $\mathrm{RP}_{\mathrm{b}}$ & $\mathrm{n}$ & $\begin{array}{c}\text { taxa } \\
\%\end{array}$ & $\mathrm{RP}_{\mathrm{b}}$ \\
\hline \multicolumn{16}{|c|}{ Idade mãe (anos) } \\
\hline$<20 * *$ & 9816 & 30,4 & 1,00 & 9839 & 32,3 & 1,00 & 9193 & 32,5 & 1,00 & 8818 & 36,0 & 1,00 & 8755 & 36,0 & 1,00 \\
\hline $20-34 * *$ & 14.380 & 50,5 & 1,66 & 14.127 & 51,9 & 1,61 & 14.370 & 53,8 & 1,66 & 14.652 & 55,2 & 1,54 & 15.447 & 57,2 & 1,59 \\
\hline \multirow[t]{2}{*}{$>35 * *$} & 681 & 77,2 & 2,54 & 718 & 72,4 & 2,24 & 711 & 77,5 & 2,39 & 795 & 77,5 & 2,15 & 828 & 80,1 & 2,23 \\
\hline & \multicolumn{3}{|c|}{$*<0,01$} & \multicolumn{3}{|c|}{$*<0,01$} & \multicolumn{3}{|c|}{$*<0,01$} & \multicolumn{3}{|c|}{$*<0,01$} & \multicolumn{3}{|c|}{$*<0,01$} \\
\hline \multicolumn{16}{|c|}{ Escolaridade mãe (anos) } \\
\hline $0-3 * *$ & 1726 & 33,9 & 1,00 & 1594 & 35,0 & 1,00 & 1236 & 36,8 & 1,00 & 914 & 45,6 & 1,00 & 847 & 44,5 & 1,00 \\
\hline 4 a $7 * *$ & 9536 & 36,0 & 1,06 & 8815 & 37,3 & 1,07 & 8546 & 38,1 & 1,04 & 7652 & 40,2 & 0,88 & 6744 & 41,8 & 0,94 \\
\hline 8 a $11 * *$ & 9588 & 45,2 & 1,33 & 9889 & 46,1 & 1,32 & 10.103 & 46,8 & 1,27 & 10.962 & 47,9 & 1,05 & 12.318 & 48,7 & 1,09 \\
\hline \multirow[t]{2}{*}{$>12 * *$} & 3722 & 61,6 & 1,82 & 4032 & 62,0 & 1,77 & 4199 & 65,2 & 1,77 & 4544 & 66,9 & 1,47 & 4979 & 68,0 & 1,53 \\
\hline & \multicolumn{3}{|c|}{$*<0,01$} & \multicolumn{3}{|c|}{$*<0,01$} & \multicolumn{3}{|c|}{$*<0,01$} & \multicolumn{3}{|c|}{$*<0,01$} & \multicolumn{3}{|c|}{$*<0,01$} \\
\hline \multicolumn{16}{|c|}{ Consultas pré-natal } \\
\hline $0-6$ ** & 12.180 & 34,4 & 1,00 & 11.410 & 35,4 & 1,00 & 9970 & 36,4 & 1,00 & 9314 & 40,4 & 1,00 & 8773 & 41,8 & 1,00 \\
\hline \multirow[t]{2}{*}{$>7 * *$} & 12.318 & 52,2 & 1,52 & 12.966 & 52,8 & 1,49 & 14.252 & 53,1 & 1,44 & 14.716 & 54,3 & 1,34 & 16.013 & 55,4 & 1,33 \\
\hline & \multicolumn{3}{|c|}{$*<0,01$} & \multicolumn{3}{|c|}{$*<0,01$} & \multicolumn{3}{|c|}{$*<0,01$} & \multicolumn{3}{|c|}{$*<0,01$} & & $<0,01$ & \\
\hline Idade gestacio & & & & & & & & & & & & & & & \\
\hline $37-41$ & 22.880 & 43,3 & 1,00 & 22.973 & 44,3 & 1,00 & 22.683 & 46,1 & 1,00 & 22.619 & 48,6 & 1,00 & 23.291 & 50,5 & 1,00 \\
\hline $22-36$ ** & 1538 & 43,3 & 1,00 & 1477 & 46,3 & 1,05 & 1414 & 49,7 & 1,08 & 1449 & 51,0 & 1,05 & 1528 & 51,7 & 1,02 \\
\hline$>42 * *$ & 316 & 50,0 & 1,15 & 249 & 59,4 & 1,34 & 169 & 55,0 & 1,11 & 213 & 57,7 & 1,19 & 139 & 57,5 & 1,14 \\
\hline & & $* 0,06$ & & & $*<0,0$ & & & $*<0,0$ & & & $*<0,01$ & & & $* 0,1$ & \\
\hline Etnia - cor da & & & & & & & & & & & & & & & \\
\hline Branca** & 23.811 & 43,4 & 1,00 & 23.610 & 45,1 & 1,00 & 23.403 & 46,7 & 1,00 & 23.368 & 49,4 & 1,00 & 24.075 & 51,0 & 1,00 \\
\hline Indígena & 53 & 33,9 & $0,78 \mathrm{~ns}$ & 70 & 38,6 & $0,85 \mathrm{~ns}$ & 48 & 35,4 & $0,76 \mathrm{~ns}$ & 51 & 29,4 & $0,60 \mathrm{~ns}$ & 55 & 30.9 & $0,61 \mathrm{~ns}$ \\
\hline Parda & 368 & 34,2 & 0,79 & 412 & 31,1 & 0,69 & 348 & 35,3 & 0,76 & 400 & 33,5 & 0,68 & 424 & 36,1 & 0,71 \\
\hline Amarela & 16 & 37,9 & $0,87 \mathrm{~ns}$ & 18 & 38,9 & $0,86 \mathrm{~ns}$ & 14 & 50,0 & $1,07 \mathrm{~ns}$ & 8 & 62,5 & $1,27 \mathrm{~ns}$ & 13 & 30,8 & $0,60 \mathrm{~ns}$ \\
\hline Preta & 483 & 43,7 & $1,01 \mathrm{~ns}$ & 475 & 39,8 & $0,88 \mathrm{~ns}$ & 429 & 43,4 & $0,93 \mathrm{~ns}$ & 375 & 41,9 & $0,85 \mathrm{~ns}$ & 374 & 45,2 & $0,89 \mathrm{~ns}$ \\
\hline Não branca & 920 & 36,3 & 0,84 & 975 & 36,0 & 0,80 & 839 & 39,7 & 0,85 & 834 & 37,3 & 0,76 & 866 & 39,6 & 0,78 \\
\hline & & $<0,01$ & & & $*<0,0$ & & & $*<0$, & & & $*<0$, & & & & 0,01 \\
\hline Total & 24.889 & 43,3 & & 24.685 & 44,7 & & 24.315 & 46,4 & & 24.294 & 48,9 & & 25.022 & 50,6 & - \\
\hline
\end{tabular}

Os dados não foram obtidos nos seguintes percentuais conforme as variáveis: idade materna (1\%); escolaridade materna (2,2\%); idade gestacional $(0,8 \%)$; pré-natal $(2,3 \%)$ e etnia/cor da pele do recém-nascido $(1,1 \%)$; *qui-quadrado: valor de $p$ para homogeneidade das taxas de cesariana; ** qui-quadrado para tendência linear no período $p<0,001$; ns= estatisticamente não significante quando o intervalo de confiança de $95 \%$ para a RPb incluia a unidade. 
Taxas e razões de prevalência de cesariana ajustadas $\left(\mathrm{RP}_{\mathrm{aj}}\right)$ para as variáveis maternas e do recém-nascido de primíparas com gravidez única e parto hospitalar. Santa Catarina, 2000 a 2004.

\begin{tabular}{|c|c|c|c|c|c|c|c|c|c|c|c|c|}
\hline \multirow[b]{2}{*}{ Variáveis } & \multicolumn{2}{|c|}{2000} & \multicolumn{2}{|c|}{2001} & \multicolumn{2}{|c|}{2002} & \multicolumn{2}{|c|}{2003} & \multicolumn{2}{|c|}{2004} & \multicolumn{2}{|c|}{$2000-2004$} \\
\hline & $\mathrm{RP}_{\mathrm{aj}}$ & IC95\% & $\mathrm{RP}_{\mathrm{aj}}$ & IC95\% & $\mathrm{RP}_{\mathrm{aj}}$ & IC95\% & $\mathrm{RP}_{\mathrm{aj}}$ & IC95\% & $\mathrm{RP}_{\mathrm{AJ}}$ & IC95\% & $\mathrm{Rp}_{\mathrm{aj}}$ & IC95\% \\
\hline \multicolumn{13}{|c|}{ Idade mãe (anos)* } \\
\hline$<20$ & 1,00 & & 1,00 & & 1,00 & & 1,00 & & 1,00 & & 1,00 & \\
\hline $20-34$ & 1,54 & $1,49-1,60$ & 1,50 & $1,45-1,55$ & 1,53 & $1,48-1,59$ & 1,42 & $1,38-1,47$ & 1,48 & $1,43-1,53$ & 1,50 & $1,47-1,52$ \\
\hline$>35$ & 2,33 & $2,21-2,45$ & 2,05 & $1,94-2,16$ & 2,18 & $2,08-2,30$ & 1,96 & $1,87-2,05$ & 2,02 & $1,93-2,11$ & 2,10 & $2,05-2,15$ \\
\hline \multicolumn{13}{|c|}{ Escolaridade da mãe anos* } \\
\hline $0-3$ & 1,00 & & 1,00 & & 1,00 & & 1,00 & & 1,00 & & 1,00 & \\
\hline 4 a 7 & 1,08 & $1,00-1,15$ & 1,07 & $1,00-1,15$ & 1,07 & $0,99-1,16$ & 0,92 & $0,86-0,99$ & 0,99 & $0,91-1,07$ & 1,16 & $1,15-1,18$ \\
\hline 8 a 11 & 1,26 & $1,18-1,36$ & 1,25 & $1,17-1,34$ & 1,25 & $1,16-1,35$ & 1,04 & $0,97-1,12$ & 1,10 & $1,02-1,19$ & 1,40 & $1,38-1,43$ \\
\hline$>12$ & 1,55 & $1,44-1,66$ & 1,52 & $1,14-1,63$ & 1,55 & $1,44-1,68$ & 1,32 & $1,23-1,53$ & 1,38 & $1,27-1,49$ & 1,50 & $1,47-1,52$ \\
\hline \multicolumn{13}{|c|}{ Consultas pré-natal ** } \\
\hline $0-6$ & 1,00 & & 1,00 & & 1,00 & & 1,00 & & 1,00 & & 1,00 & \\
\hline$>7$ & 1,34 & $1,31-1,39$ & 1,33 & $1,29-1,37$ & 1,28 & $1,24-1,32$ & 1,21 & $1,18-1,25$ & 1,19 & $1,15-1,22$ & 1,27 & $1,26-1,29$ \\
\hline \multicolumn{13}{|c|}{ Idade gestacional * } \\
\hline $37-41$ & 1,00 & & 1,00 & & 1,00 & & 1,00 & & 1,00 & & 1,00 & \\
\hline $22-36$ & 1,07 & $1,01-1,15$ & 1,13 & $1,05-1,23$ & 1,14 & $1,06-1,23$ & 1,09 & $1,02-1,17$ & 1,06 & $0,99-1,13$ & 1,10 & $1,06-1,13$ \\
\hline$>42$ & 1,17 & $1,03-1,34$ & 1,07 & $1,02-1,15$ & 1,35 & $1,18-1,55$ & 1,27 & $1,06-1,51$ & 1,16 & $1,01-1,35$ & 1,22 & $1,14-1,30$ \\
\hline \multicolumn{13}{|c|}{ Etnia/cor da pele* } \\
\hline Branca & 1,00 & & 1,00 & & 1,00 & & 1,00 & & 1,00 & & 1,00 & \\
\hline Indígena & 1,01 & $0,70-1,47$ & 1,11 & $0,83-1,49$ & 1,06 & $0,73-1,53$ & 0,72 & $0,47-1,10$ & 0,86 & $0,58-1,27$ & 0,79 & $0,75-0.85$ \\
\hline Parda & 0,86 & $0,74-0,99$ & 0,76 & $0,65-0,87$ & 0,85 & $0,73-0,98$ & 0,76 & $0,66-0,87$ & 0,77 & $0,68-0,87$ & 0,95 & $0,82-1,12$ \\
\hline Amarela & 0,95 & $0,85-1,016$ & 0,94 & $0,84-1,05$ & 1,00 & $0,89-1,11$ & 0,94 & $0,84-1,05$ & 0,94 & $0,84-1,05$ & 0,95 & $0,90-1,00$ \\
\hline Preta & 1,21 & $0,68-2,16$ & 0,95 & $0,55-1,67$ & 1,09 & $0,61-1,03$ & 1,48 & $0,91-2,41$ & 0,63 & $0,28-1,38$ & 1,02 & $0,78-1.33$ \\
\hline Não Branca & 0,94 & $0,87-0,98$ & 0,88 & $0,83-0,97$ & 0,94 & $0,89-1,05$ & 0,86 & $0,79-0,93$ & 0,87 & $0,75-0,94$ & 0,91 & $0,88-0,96$ \\
\hline
\end{tabular}

1,00: categoria de referência para cálculo da RPaj; idade gestacional: em semanas; * modelo ajustado para todas as variáveis na tabela exceto pré-natal ; ** modelo ajustado para todas as variáveis na tabela.

\section{Discussão}

As altas taxas de cesariana encontradas neste estudo, assim como o aumento dessas no período, estão de acordo com o apresentado por outros autores no Brasil. ${ }^{10,17,19,22}$

As taxas para o Estado de Santa Catarina estão acima da média nacional e são o triplo do preconizado pela WHO. ${ }^{7}$ O perfil encontrado para o estado concorda com aquele descrito para outras regiões brasileiras com semelhante nível de desenvolvimento socioeconômico. ${ }^{10,19,22}$ Um aumento importante das taxas de cesariana no período estudado é preocupante, uma vez que aponta para a má utilização da tecnologia médica no parto.
A associação positiva entre grau de escolaridade materna e taxa de cesariana, descrita pelo estudo, sugere uma estreita relação entre o uso do procedimento, poder aquisitivo e acesso aos serviços, conforme já relatado em outros estudos. ${ }^{10,22}$

Estes últimos resultados trazem à tona o princípio da "equidade inversa", conforme postulado por Victora et al., ${ }^{20}$ com maiores taxas de cesariana entre mulheres com maior escolaridade, potencialmente aquelas menos expostas aos fatores de risco para parto cirúrgico.

Por outro lado, ao comparar os extremos do período, a redução nas razões de prevalência encontrada para quase todas as categorias estudadas, remete a uma redução nos efeitos do mesmo fenômeno, 
provável reflexo de um maior acesso à tecnologia no parto entre as camadas de menor padrão socioeconômico, pelo menos em parte atribuível a uma maior liberalidade da prática obstétrica, incluindo uma ampliação das indicações médicas.

O reduzido número de nascidos vivos na população indígena, para cada um dos anos, aponta para a necessidade de futuros estudos que visem explorar as relações entre as diferenças étnicas e o uso da tecnologia. Entretanto, os resultados encontrados ao analisar os recém-nascidos da categoria étnica "não branca" como um grupo único indicam a existência de um efeito protetor para a ocorrência de cesariana entre as populações etnicamente menos privilegiadas.

Quando não clinicamente indicada, a cesária se mostra menos segura que o parto normal. ${ }^{8}$ Como qualquer outra cirurgia, o parto cesário não está livre das possíveis complicações anestésicas, acidentes operatórios, problemas relacionados com transfusão sanguínea, entre outros, além dos problemas que a fragilidade física acarreta nos cuidados com o recémnascido. ${ }^{23}$ Nesse contexto, os resultados do presente estudo, mostrando uma tendência crescente nas taxas de cesariana entre mães com nível socioeconômico mais baixo, teoricamente com maior risco obstétrico, tornam o quadro ainda mais preocupamente.

As altas taxas encontradas entre as mulheres de idade igual ou superior a 35 anos, quando comparadas às adolescentes, concorda com resultados de outros estudos ${ }^{10,24,25}$ que apontam "gestação tardia" como fator associado à cesariana. Ainda, uma maior prevalência do procedimento entre mães de recémnascidos prematuros aponta para a ocorrência de "casualidade reversa", onde a utilização excessiva da cesariana como cirurgia eletiva estaria levando a maiores índices de prematuridade e não vice-versa, também relatado em estudo semelhante conduzido no Rio Grande do Sul. ${ }^{10}$

Resultados apontando para um aumento crescente nas taxas de cesariana entre os recém-nascidos prétermo estão em sintonia com outros estudos recentes cujos resultados trazem à tona a questão da intervenção em prematuros e dos riscos associados às cesarianas eletivas. 5,6

Há algum tempo o Brasil convive com altas taxas de cesariana e poucas foram as ações dirigidas ao problema e as estratégias adotadas mostraram-se pouco efetivas. ${ }^{19}$ Acredita-se que o fracasso dessas intervenções possa estar, pelo menos parcialmente, associado à cultura da cesária no país, já amplamente debatida como parte importante no contexto das altas taxas. $^{21}$

A sentença "uma vez cesária, sempre cesária", preconizada por Gragin, em 1916, conforme Cunningham et al., ${ }^{1}$ fez com que o antecedente de cesariana se consolidasse historicamente como fator de risco para uma nova cesariana. Ainda que os dados disponíveis pelo Sistema de Informação sobre Nascidos Vivos (SINASC) não permitam avaliar o papel das cesarianas prévias, a investigação dos fatores associados às taxas de cesariana entre as primíparas, conduzida no presente estudo, ganha importância no contexto das cesárias de repetição, uma vez que esse grupo pode ser visto como primordial quando ações dirigidas a uma diminuição do abuso da tecnologia no parto são planejadas.

\section{Referências}

1. Cunningham GF, Mac Donald PC, Leveno PC. Williams Obstetrics. 18th ed. Stamford: Appleton \& Lange; 1997.

2. Faúndes A, Cecatti JG. Which policy for caesarean sections in Brazil? An analysis of trends and consequences. Health Policy Plan. 1993; 8: 33-42.

3. Barros FC, Vaughan JP, Victora CG, Huttly S. Epidemic of caesarean sections in Brazil. Lancet. 1991; 338: 167-9.

4. Yang H, Kramer MS, Platt RW, Blondel B, Breart G, Morin I, Wilkins R, Usher R. How does early ultrasound scan estimation of gestational age lead to higher rates of preterm birth? Am J Obstet Gynecol. 2002; 186: 433-7.

5. Barros FC, Victora CG, Barros AJ, Santos IS, Albernaz E, Matijasevich A, Domingues MR, Sclowitz IK, Hallal PC, Silveira MF, Vaughan JP. The challenge of reducing neonatal mortality in middle-income countries: findings from three Brazilian birth cohorts in 1982, 1993, and 2004 Lancet. 2005; 365: 847-54.
6. Villar J, Wojdyla D, Zavaleta N, Carroli G, Velazco A, Shah A, Campodonico L, Bataglia V, Faundes A, Langer A, Narvaez A, Donner A, Romero M, Reynoso S, de Padua KS, Giordano D, Kublickas M, Acosta A, WHO 2005 Global Survey on Maternal and Perinatal Health Research Group. Caesarean delivery rates and pregnancy outcomes: the 2005 WHO global survey on maternal and perinatal health in Latin America. Lancet. 2006; 367: 1819-29.

7. WHO (World Health Organization). Appropriate technology for birth. Lancet. 1985; 2: 436-7.

8. Belizan JM, Althabe F, Barros FC, Alexander S. Rates and implications of caesarean sections in Latin America: ecological study. Br Med J. 1999; 319: 1397-102.

9. Roosmalen JV, Does CDV. Caesarean birth rates worldwide. Trop Geogr Med. 1995; 47: 19-22.

10. Freitas PF, Drachler ML, Leite JCC, Grassi PR. Desigualdade social nas taxas de cesariana em primíparas no Rio Grande do Sul. Rev Saúde Pública. 2005; 39: 761-7. 
11. SINASC (Sistema de Informações sobre Nascidos Vivos). Santa Catarina. Arquivos de declarações de nascidos vivos. Disponível em URL: http://tabnet.datasus.gov.br/ tabdata/sinasc/dados /nov_indice.htm [2006 jan 5]

12. Kristensen MO, Hedegaard M, Secher NJ. Can the use of caesarean section be regulated? A review of methods and results. Acta Obstet Gynecol Scand. 1998; 77: 951-60.

13. Walker R, Turnbull D, Wilkinson C. Strategies to address global caesarean section rates: a review of the evidence. Birth. 2002; 29: 28-39.

14. Althabe F, Belizan JM, Villar J, Alexander S, Bergel E, Ramos S, Romero M, Donner A, Lindmark G, Langer A, Farnot U, Cecatti JG, Carroli G, Kestler E, Latin-American Caesarean Section Study Group. Mandatory second opinion to reduce rates of unnecessary caesarean sections in LatinAmerica: a cluster randomised controlled trial. Lancet. 2004; 363: 1934-40.

15. Gomes UA, Silva AAM, Bettiol H, Barbieri MA. Risk factors for the increasing caesarean section rate in Southeast Brazil: a comparison of two birth cohorts, 1978-1979 and 1994. Int J Epidemiol. 1999; 28: 687-94.

16. Barros FC, Vaughan JP, Victora CG. Why so many caesarean sections? The need for a further policy change in Brazil. Health Policy Plan. 1986; 1: 19-29.

17. Potter JE, Berquó E, Perpétuo IHO, Leal OF, Hopkins K, Souza MR. Unwanted caesarean sections among public and private patients in Brazil: prospective study. Br Med J. 2001; 323: $1155-8$.
18. Béhague DP, Victora CG, Barros FC. Consumer demand for caesarean sections in Brazil: population based birth cohort study linking ethnographic and epidemiological methods. $\mathrm{Br}$ Med J. 2002; 324: 942-5.

19. Silveira DS, Santos IS. Fatores associados à cesariana entre mulheres de baixa renda em Pelotas, Rio Grande do Sul, Brasil. Cad Saúde Pública. 2004; 20: 231-41.

20. Victora CG, Vaughan JP, Barros FC, Silva AC, Tomasi E. Explaining trends in inequities: evidence from Brazilian child health studies. Lancet. 2000; 356: 1093-8.

21. Freitas PF, Behague D. Brazil's culture of caesarean births. Health Exchange. 1995; 10: 8-9.

22. Moraes MS, Goldenberg P. Cesárias: um perfil epidêmico. Cad Saúde Pública. 2001; 17: 509-19.

23. Resende J. Obstetrícia, conceito, propósitos, súmula histórica. In: Resende J, organizador. Obstetrícia. 8. ed. Rio de Janeiro: Guanabara; 1987.

24. Cabral SA, Costa CF, Cabral SF. Correlação entre a idade materna, paridade, gemelaridade, síndrome hipertensiva e ruptura prematura de membranas e a indicação de parto cesáreo. Rev Bras Ginecol Obstet. 2003; 25: 739-44.

25. Scholz HS, Haas J, Petru E. Do primíparas aged 40 years or older carry an increased obstetric risk? Prev Méd. 1999; 29: 263-6.
Recebido em 6 de janeiro de 2006

Versão final apresentada em 29 de agosto de 2007

Aprovado em 3 de setembro de 2007 